\title{
Effects of Electrostatic Discharge Stress on Current- Voltage and Reverse Recovery Time of Fast Power Diode
}

\author{
Daoheung Bouangeune ${ }^{1}$, Sang-Sik Choi ${ }^{2}$, Deok-Ho Cho ${ }^{2}$, Kyu-Hwan Shim ${ }^{1,2}$, Sung-Yong Chang ${ }^{3}$, \\ See-Jong Leem ${ }^{4, *}$, and Chel-Jong Choi ${ }^{1, *}$
}

Abstract-Fast recovery diodes (FRDs) were developed using the $\mathrm{p}^{++} / \mathrm{n}^{-} / \mathrm{n}^{++}$epitaxial layers grown by low temperature epitaxy technology. We investigated the effect of electrostatic discharge (ESD) stresses on their electrical and switching properties using current-voltage (I-V) and reverse recovery time analyses. The FRDs presented a high breakdown voltage, $>450 \mathrm{~V}$, and a low reverse leakage current, $<10^{-9}$ A. From the temperature dependence of thermal activation energy, the reverse leakage current was dominated by thermal generation-recombination and diffusion, respectively, at low and high temperature regions. By virtue of the abrupt junction and the $P t$ drive-in for the controlling of carrier lifetime, the soft reverse recovery behavior could be obtained along with a well-controlled reverse recovery time of $\mathbf{2 1 . 1 2}$ ns. The FRDs exhibited excellent ESD robustness with negligible degradations in the $\mathrm{I}-\mathrm{V}$ and the reverse recovery characteristics up to $\pm 5.5 \mathrm{kV}$ of $\mathrm{HBM}$ and $\pm 3.5 \mathrm{kV}$ of IEC61000-4-2 shocks. Likewise, transmission line pulse (TLP) analysis reveals that the FRDs can handle the maximum peak pulse current, $I_{p p, \max }$, up to $30 \mathrm{~A}$ in the forward mode and down to -

Manuscript received May. 18, 2014; accepted Jul. 4, 2014

${ }^{1}$ School of Semiconductor and Chemical Engineering, Semiconductor Physics Research Center (SPRC), Chonbuk National University, Jeonju 561-756, Republic of Korea.

${ }^{2}$ R\&D Division, Sigetronics, Inc., Jeonju 561-756, Republic of Korea

${ }^{3}$ Power Integrity Group, Korea Electric Power Research Institute (KEPRI), Daejeon 305-380, Republic of Korea.

${ }^{4}$ Department of Hydrogen Energy, Dongshin University, Naju 520-714, Republic of Korea.

E-mail : leemsj11@naver.com,cjchoi@jbnu.ac.kr
$24 \mathrm{~A}$ in the reverse mode. The robust ESD property can improve the long term reliability of various power applications such as automobile and switching mode power supply.

Index Terms-ESD, IEC 61000-4-2, TLP, fast recovery diode, carrier transport mechanism

\section{INTRODUCTION}

Fast recovery power diodes (FRDs) are the main part of power electronic circuits, especially, power switching circuits. In switching mode, FRDs act as a freewheeling device to protect the power semiconductors against high voltage spike damage, which is usually generated from inductance loads such as motor coils or relay windings suddenly turned "OFF". However, the FRD itself could not avoid degradation or failure due to high voltage spike, snappy recovery and electrostatic discharge (ESD) [1-4]. The $p-n$ junction is the main structure of the FRDs, and is inevitable from the effects of ESD during the fabrication, testing, packaging and printing to board circuit process. Thus, the ESD can give a significant effect on the reliability of FRDs. Moreover, FRDs operate at high breakdown voltage, are normally more susceptible to ESD failures due to the power limited (low reverse saturation currents and high resistance value). The ESD is known as a high transient current and voltage source with a short duration time less than $150 \mathrm{~ns}$, which is originated from the transfer of electrostatic charges between two objects with different electrical potentials 
[5]. High transient voltages generated by the ESD can lead to the burn-out of the metal contact edge, surface, $\mathrm{Si}^{-\mathrm{SiO}_{2}}$ interface, and p-n junction [2]. Typical damages are known as the defect states, which are the main sources of the leakage current [2,6] and noise [7] (trapping and de-trapping of carriers in defect states, especially, at the $\mathrm{Si}_{-} \mathrm{SiO}_{2}$ interface). This may also result in the degradation of the device performance as well as electrical and switching properties like the reverse leakage current, blocking voltage and reverse recovery time. Hence, the main issues involved with the effects of ESD stress on electrical and switching properties need to be addressed to guide a way of improving long-term reliability. However, almost no study is focused on investigating the effects of ESD stress on switching properties for high power FRDs. Very recently, it was reported that the Si transient voltage suppression (TVS) diodes achieved small dynamic resistance, low reverse leakage current and excellent ESD performance in excess of the IEC standard requirement $( \pm 8 \mathrm{kV}$ in contact discharge) [8-10].

In this work, Si FRDs were fabricated using low temperature epitaxy technology. Then, the influence of ESD stress on their electrical properties such as the current-voltage (I-V) and reverse recovery time $\left(\mathrm{t}_{\mathrm{rr}}\right)$, were investigated in accordance with various ESD test including HBM, IEC61000-4-2 (IEC) and transmission line pulse (TLP) analysis. It will be shown that the manufactured FRDs are effective in ESD performance in conjunction with the I-V and the reverse recovery characteristics.

\section{Experimental Procedure}

Fig. 1 shows the schematic of the proposed FRD with the $\mathrm{p}^{++} / \mathrm{n}^{-}$junction structure formed on the heavily doped n-type Si (100) substrate. First, the Si wafer was cleaned using an HF solution in order to remove impurities and native oxides. After inserting the cleaned $\mathrm{Si}$ wafer into a reduced-pressure chemical vapor deposition (RPCVD) chamber via a nitrogen purged load-lock chamber, it experienced high temperature hydrogen baking at $950{ }^{\circ} \mathrm{C}$ for $5 \mathrm{~min}$. Then the $\mathrm{n}$ - type $\mathrm{Si}$ epitaxial layers were grown using doping gases of phosphine $\left(\mathrm{PH}_{3}\right)$. The epitaxy process was performed at low temperature (around $600-800{ }^{\circ} \mathrm{C}$ ) to minimize the diffusion of dopant atoms and possible degradation of device performance

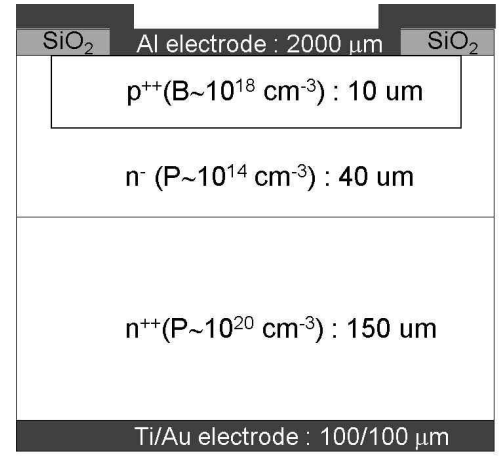

Fig. 1. Cross-section view of the proposed FRD.

caused by the non-uniform local electric field concentrated at $\mathrm{SiO}_{2} / \mathrm{Si}$ interfaces. Linearly-graded junctions are frequently formed at high temperature for conventional drive-in processes. For forming the emitter layer, the boron implantation with a dose of $5 \times 10^{14} / \mathrm{cm}^{2}$ was employed. In order to control the minority carrier lifetime of FRD, Pt atoms were diffused from $10 \mathrm{~nm}$ thick Pt film deposited on back side $\mathrm{n}++$-substrate for 40 min at $900-950{ }^{\circ} \mathrm{C}$, followed by removal of excess $\mathrm{Pt}$ layer. As the top electrode contact, a $2000 \mathrm{~nm}$-thick $\mathrm{Al}$ film was deposited by means of an e-beam evaporator through the square shaped pattern with a size of $2.2 \times 2.2$ $\mathrm{mm}^{2}$. The back side of substrates was grinded down to $130 \mu \mathrm{m}$ in thickness, and followed by $100 / 500 \mathrm{~nm}$-thick $\mathrm{Ti} / \mathrm{Au}$ films for the back electrode. Current-voltage (I-V) curves and breakdown voltage measurements were performed using a parameter analyzer (Agilent 4156C) and Sony/Textronix 370 curve tracer, respectively. The ESD properties were analyzed using an ESD simulator, NoiseKenESS-6008, which can supply an output voltage of up to $\pm 8 \mathrm{kV}$ in a human body model (HBM) and an ESS-2000 with a discharge gun TC-815R was used for the IEC61000-4-2 standard analyses. Meanwhile, TLP property was analyzed using the Barth TLP 4002 system, where the high-voltage pulse was applied step-by-step with 2 ns rise time and 100ns pulse-width. The reverse recovery characteristics were measured using the circuit diagram as shown in Fig. 2, which consists a Gwinstek GPR-30H10 and Tektronix AFG3102 as a DC power supply and pulse generation for controlling "OFF" and "ON" state of the n-channel MOSFET, respectively. The reverse recovery waveforms were captured using oscilloscope Tektronix DPO7104 equipped with TCP0030 current probe. 


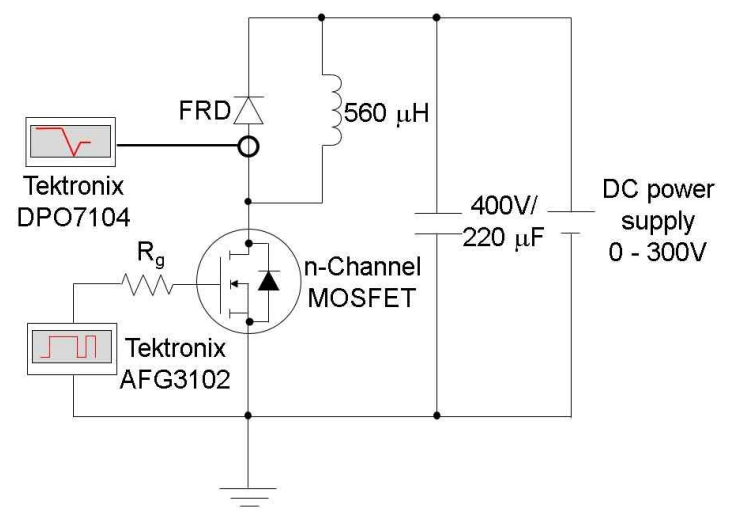

Fig. 2. Schematic for the reverse-recovery characterization circuit.

\section{RESULTS AND DISCUSSION}

The proposed FRDs rated to $400 \mathrm{~V}$ (avalanche breakdown at $450 \mathrm{~V}$ ) were controlled by doping concentration and epi-layer thickness of the base layer. Generally, a high breakdown voltage diode requires wide and light base doping layer, whereas it also increases the recombination lifetime, lead to slow recovery time. In our case, we controlled the minority carrier lifetime by using the $\mathrm{Pt}$ diffusion instead the gold due to it implements lower temperature dependent on the reverse leakage current, fast recovery at high current operation [11] and softer recovery [12]. The Pt diffusion introduces the defect states in the band gap, acting as the minority carrier lifetime killer, which accomplish fast recovery time. The reverse recovery time, $t_{r r}$ is the most important parameters of the power diode and general used as switching speed monitor, which defined as the total time require for depleting the storage charge at the $p-n$ junction during turned "OFF" state. Fig. 3 plots the current and voltage waveforms captured during diode turning "off state" at various the $d I_{F} / d t$ rate with controlling $I_{F}=1 \mathrm{~A}$. Notable, increasing the $d I_{F} / d t$ rate significantly decreases the $t_{r r}$ from $24.8 \mathrm{~ns}$ and $14.6 \mathrm{~ns}$ while the $d I_{F} / d t$ rate varies from $35 \mathrm{~A} / \mu$ s to $280 \mathrm{~A} / \mu \mathrm{s}$. However, undesirable the peak reverse current $\left(\mathrm{I}_{\mathrm{RRM}}\right)$ increases and snappiness occur.

In order to estimate the exact $t_{r r}$ value, $V_{R}=30 \mathrm{~V}$ and $I_{F}=1 \mathrm{~A}$ with controlling $d I_{F} / d t=100 \mathrm{~A} / \mu \mathrm{s}$, are widely uses to estimate the $t_{r r}$ values of the diode in the semiconductor industries [13, 14]. As shown in Fig. 4, the reverse recovery waveform, was consistent of
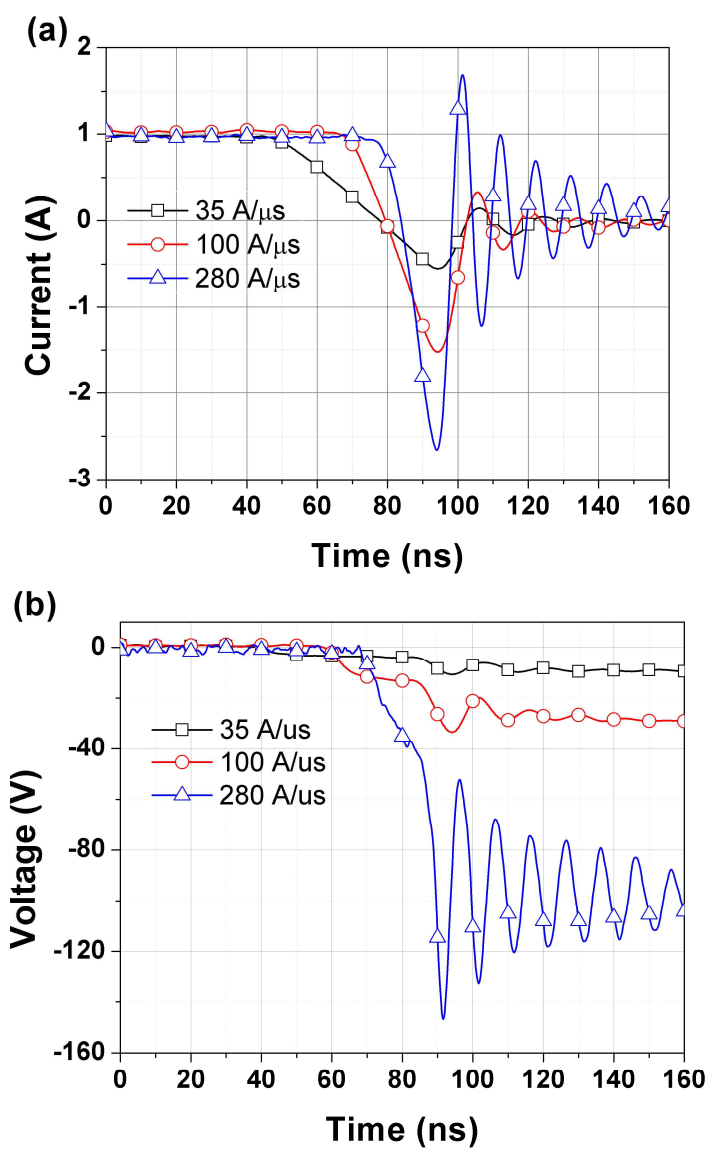

Fig. 3. (a) Current, (b) voltage waveforms captured from the FRDs at various $d I_{F} / d t$ rate of 35,100 and $280 \mathrm{~A} / \mu \mathrm{s}$.

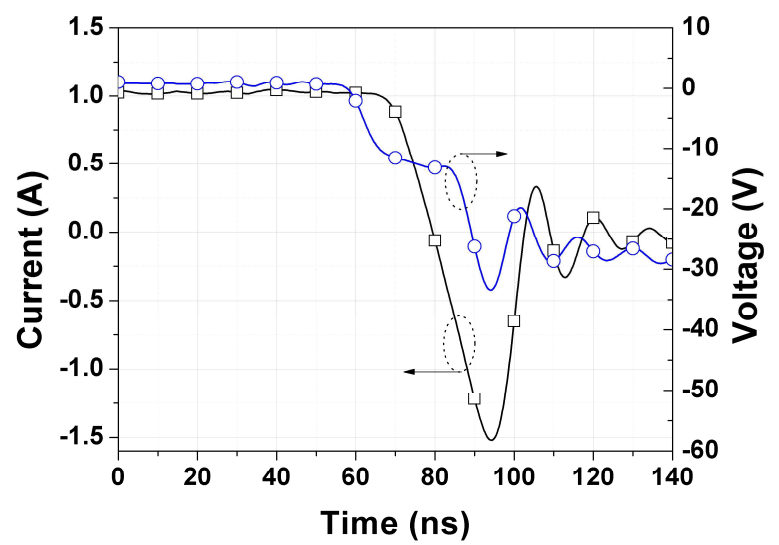

Fig. 4. Current and voltage waveforms of a FRD measured at $V_{R}=30 \mathrm{~V}$ and $I_{F}=1$ A with controlling $d I_{F} / d t=100 \mathrm{~A} / \mathrm{us}$.

negligible oscillation, this normally observe in the diode with short minority carrier lifetime as well as $t_{r r}$ [15]. The $t_{r r}$ and $I_{R R M}$ were $21.12 \mathrm{~ns}$ and $-1.54 \mathrm{~A}$, respectively. Even the oscillation produced the overshot voltage of $33.3 \mathrm{~V}$ or 
$1.11 \times V_{R}$, however, it was considered as the very fast and soft reverse recovery $\mathrm{FRD}$ when the soft factor, $\mathrm{S}$ (defined as the ratio of falling time $\left(t_{f}\right)$ and storage time $\left(t_{s}\right)$ [12]), was 0.6. A short $t_{r r}$ with soft reverse recovery waveform minimizes the generation of unwanted EMI and large voltage spikes in the system.

Fig. 5 shows the $\mathrm{I}-\mathrm{V}$ characteristics of a FRD at various temperatures in ranging from $27{ }^{\circ} \mathrm{C}$ to $150{ }^{\circ} \mathrm{C}$, the reverse current, $I_{R}$ increases as the rising operating temperature when the carriers in semiconductor materials are followed the Boltzmann statistics. However, the reverse leakage current was kept at less $10^{-5} \mathrm{~A}$.

The temperature dependence of the reverse current as given in Fig. 5, has provided a useful for investigation the effect of the $\mathrm{Pt}$ diffusion on the reverse leakage current conduction mechanism. For the one-side abrupt junction, the reverse current given by the Shockley equation can be expressed as [16]

$$
I_{R}=S q \sqrt{\frac{D_{p}}{\tau_{p}}} \frac{n_{i}^{2}}{N_{D}}+\frac{e S W n_{i}}{\tau_{g}}
$$

where $S$ is the p-n junction area, $N_{D}$ is the base doping concentration, $W$ is the depletion region width, $D_{p}$ is the diffusion coefficient of hole, $\tau_{p}$ is the mean hole recombination lifetime and $\tau_{g}$ is the mean time to generate electron-hole pairs. Since $n_{i} \sim \exp \left(-E_{g} / 2 k_{B} T\right)$, the temperature dependence of the exponential terms is more stronger to than other terms. Then, the Eq. (1), can be rewritten as

$$
I_{R} \sim \exp \left(\frac{E_{g}}{k_{B} T}\right)+\exp \left(\frac{E_{g}}{2 k_{B} T}\right)
$$

The slope of $\ln I_{R}-1 / k_{B} T$ plot is defined as the thermal activation energy $\left(E_{a}\right)$ of reverse current. In principle, $E_{a}$ should be close to the $\mathrm{Si}$ band gap $\left(E_{g}\right)$ or $E_{g} / 2$ in diffusion- or generation-recombination dominated regimes, respectively. It is well known that the generation-recombination current is mainly attributed to the carriers generated in the depletion region and dominate at low temperature. Meanwhile, the diffusion current is caused by the diffusion of minority carriers approaching the depletion edge and dominates at high temperature. The $E_{a}$ values, determined from the slop of

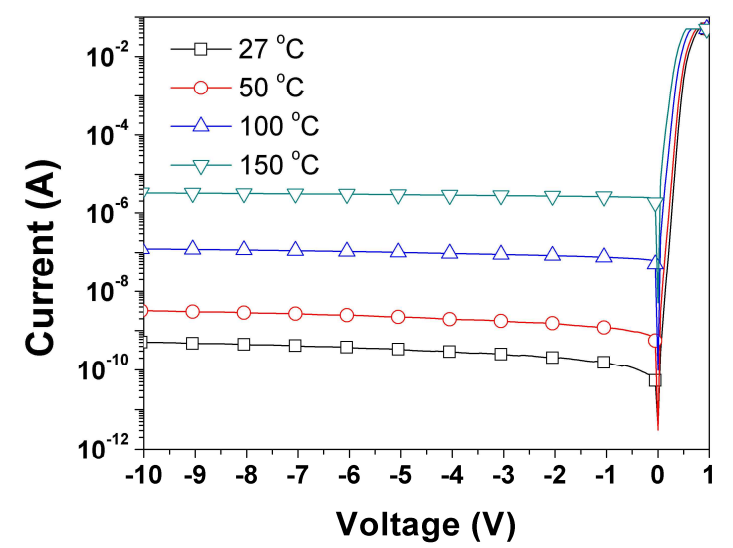

Fig. 5. I-V characteristics of a FRD measured at various temperatures ranging from $27^{\circ} \mathrm{C}$ to $150{ }^{\circ} \mathrm{C}$.

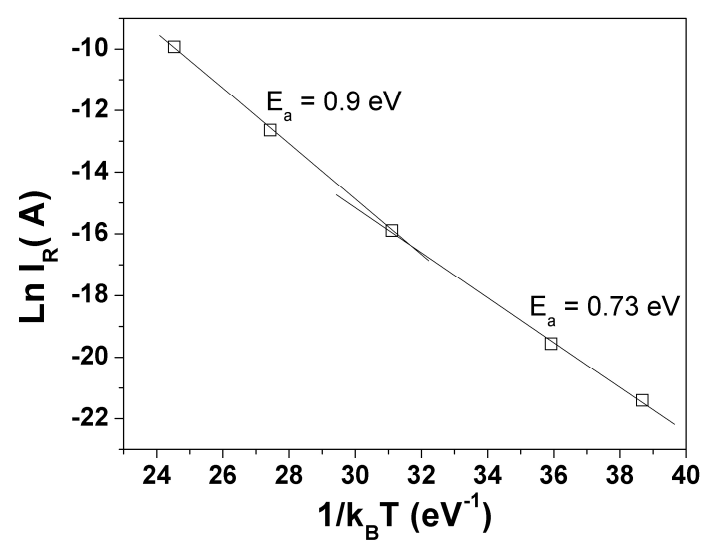

Fig. 6. Plot of $\ln I_{R}$ vs. $1 / k_{B} T$ at reverse bias of $10 \mathrm{~V}$ for obtaining the thermal activation energy of reverse leakage current.

$\ln I_{R}-1 / k_{B} T$ plot, were found to be 0.73 and $0.9 \mathrm{eV}$ at low and high temperatures, respectively. This implies that the Pt diffusion induces the acceptor levels around $0.20 \sim 0.37 \mathrm{eV}$ below the conduction band of the base Si epi-layer. Pt-induced acceptor levels calculated here are comparable to the values which were reported in a previous work [11]. Typically, the acceptor levels contribute greatly path for leakage current. However, $E_{a}$ values were nearly equal to $E_{g} / 2$ and $E_{g}$ of $\mathrm{Si}$, indicating the reverse leakage current of a proposed FRD was followed in principle as generation-recombination dominated at low temperature and diffusion current dominated at high temperature, which normal observe in the general p-n junction diodes.

For investigating the effect of ESD stress on the electrical and switching properties such as the reverse leakage current, breakdown voltage and $t_{r r}$, the FRDs 
(a)

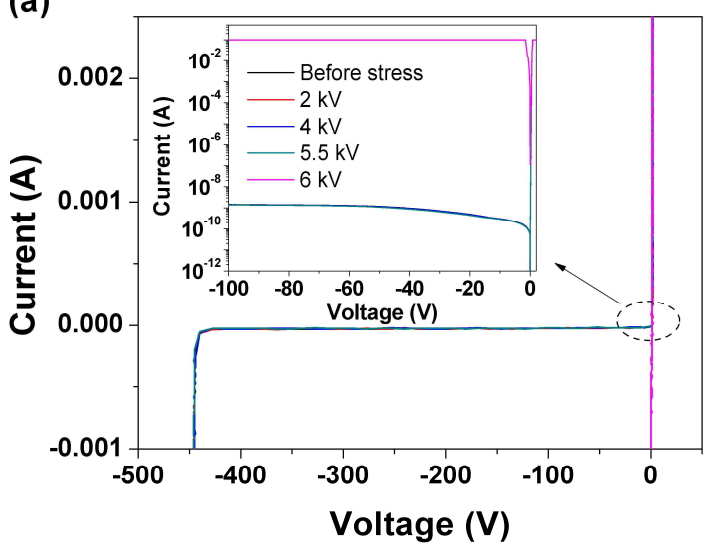

(b)

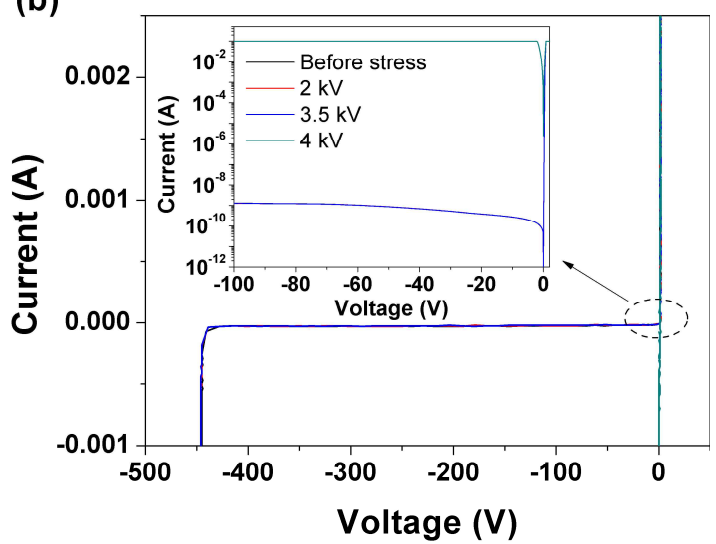

Fig. 7. I-V characteristics of the FRDs measured before and after experiencing various ESD stresses (a) HBM, (b) IEC 61000-4-2.

were also subjected to various levels of ESD pulse of 10 discharges in both positive and negative polarity with one sec time intervals. DC I-V curves and reverse recovery waveforms were measured at initially, and every time of the samples after experiencing ESD shocks to monitor the device failure. As the results, Figs. 7(a) and (b) show the I-V curves of the FRDs before and after the HBM and IEC testing, respectively. It is observed that the device was capable of withstanding up to $\pm 5.5 \mathrm{kV}$ of $\mathrm{HBM}$ and $\pm 3.5 \mathrm{kV}$ of IEC without degradations in I-V curves. However, the failure happened after $\pm 6 \mathrm{kV}$ of $\mathrm{HBM}$ and $\pm 4 \mathrm{kV}$ of IEC stressed, which observed from rapid increasing of the reverse leakage current.

Similar to the ESD stress-dependent I-V characteristics as shown in Fig. 7, the reverse recovery waveforms captured from FRDs remained unchangeable even after $\pm 5.5 \mathrm{kV}$ of HBM and $\pm 3.5 \mathrm{kV}$ of IEC stresses as shown in Figs. 8(a) and (b), respectively. On the other hand, after $\pm 6 \mathrm{kV}$ of HBM and $\pm 4 \mathrm{kV}$ of IEC applied, the
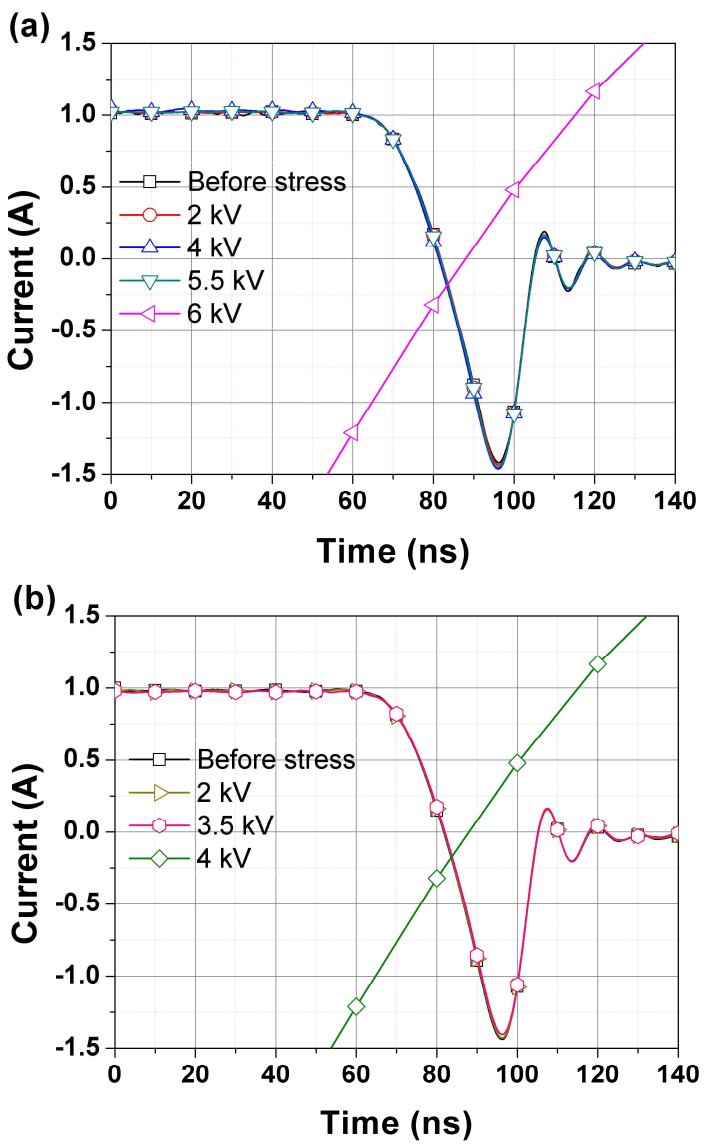

Fig. 8. Reverse recovery waveform of the FRDs measured before and after experiencing various ESD stresses (a) HBM, (b) IEC 61000-4-2.

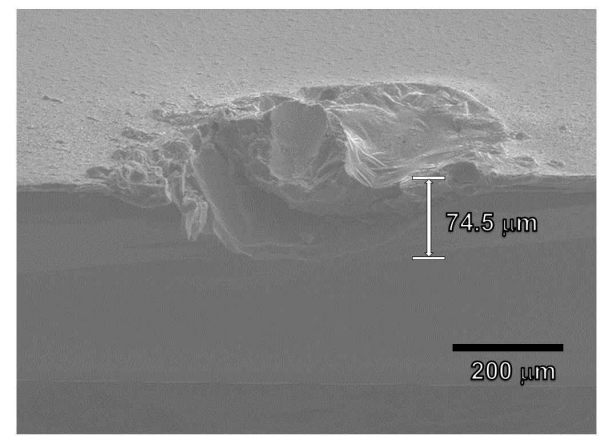

Fig. 9. SEM images showing the damaged region by $\pm 4 \mathrm{kV}$ IEC shocks.

devices were loosed the switching property, which correspond to the failure in $\mathrm{I}-\mathrm{V}$ characteristics as shown in Figs. 7(a) and (b).

The experiment above demonstrates that the FRD cannot avoid hard breakdown and failure at high pulse voltage stress. The scanning electron microscope (SEM) picture was given in Fig. 9, reveals the cross-section 


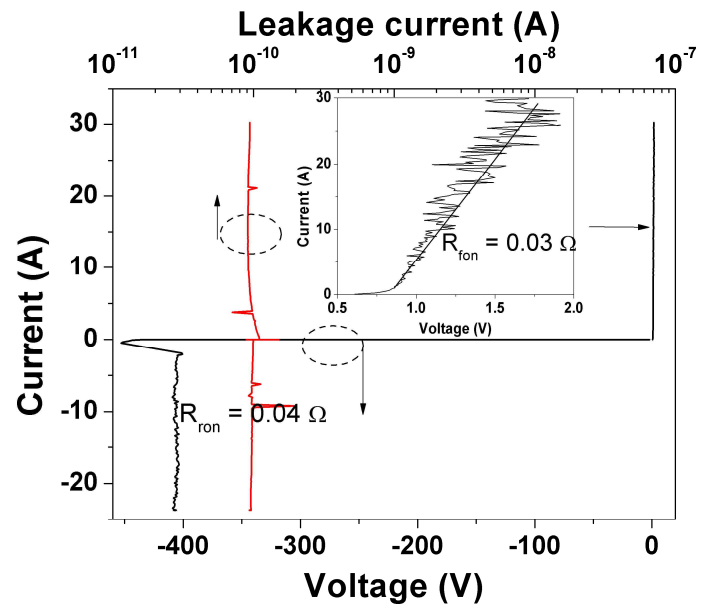

Fig. 10. TLP I-V characteristics measured from a proposed FRD.

surface of a FRD damaged by $\pm 4 \mathrm{kV}$ of IEC shocks. The ESD shocks burnout the metal electrode and p-n junction with the depth of $74.5 \mu \mathrm{m}$, which could be responsible for the device failure.

Furthermore, its ESD performance was also confirmed by TLP analysis, which known as the standard ESD test in the semiconductor industries [17-20]. The TLP test results as shown in Fig. 10, reveals that the FRD was triggered $\mathrm{ON}$ at $0.7 \mathrm{~V}$ and $-450 \mathrm{~V}$ for the positive and negative TLP polarity, the turn-on dynamic resistance $\left(R_{O n}\right)$ obtained from a linear region were $0.03 \Omega$ and 0.04 $\Omega$. Typical small $R_{O n}$ value has an advantage in minimizing Joule heating, which is the major cause of device degradation and failure. The FRD could handle current up to $-24 \mathrm{~A}$ for the negative TLP polarity and over 30 A for the positive polarity, while maintaining the reverse leakage current level as well as the original values.

As discussed above, we investigated versatile properties of the new FRDs for the first time in terms of ESD, I-V, switching characteristics. The influence of various ESD models stresses on $\mathrm{I}-\mathrm{V}$ characteristics and switching properties guarantee that fabricated FRDs are high reliability and very strong ESD robustness. We believe it is crucial to address those properties for power semiconductor devices, especially in order to secure long-term reliability in harsh environments and to avoid possible serious failures.

\section{CONCLUSION}

We have developed a new epitaxial FRD to be operable up to $400 \mathrm{~V}$ while maintaining very small reverse leakage current $<10^{-9} \mathrm{~A}$. The experimental data demonstrated very strong ESD robustness for the FRD to maintain in conjunction with excellent performance in I$\mathrm{V}$ and switching properties. The abrupt dopant profile in the region of $\mathrm{p}^{++} \mathrm{n}$ junction could result in the soft reverse recovery as assisted by the reduction in carrier lifetime controlled to $21.12 \mathrm{~ns}$. The FRD presented ESD protection capability as strong as $\pm 3.5 \mathrm{kV}$ of IEC61000$4-2, \pm 5.5 \mathrm{kV}$ of HBM, and the $I_{p p \text {, } \max }$ as measure in the TLP analysis $>30 \mathrm{~A}$ in the forward mode and $-24 \mathrm{~A}$ in the reverse mode. The ESD strength may confirm useful guidelines about the long term reliability of switch power supply to be assembled with power transistors like IGBT or Power MOSFETs.

\section{ACKNOWLEDGMENTS}

This research was supported by the Basic Science Research Program through the National Research Foundation of Korea (NRF) funded by the Ministry of Education (2009-0094031), Priority Research Centers Program (2011-0031400) funded by the Ministry of Education, Republic of Korea and it was also supported by the Future Semiconductor Device Technology Development Program (Grant No. 10044651) funded By MOTIE (Ministry of Trade, Industry \& Energy) and KSRC (Korea Semiconductor Research Consortium).

\section{REFERENCES}

[1] M. T. Rahimo, and N. Y, A Shammas, "Reverse recovery failure modes in modern fast recovery diodes," International Conference on Microelectronic, Vol.2, pp.659-662, May, 2000.

[2] M. Diatta et al, "Understanding the failure mechanisms of protection diodes during system level ESD: toward repetitive stress robustness", IEEE Trans. Electron Devices. Vol.59, pp.108-113, January, 2012

[3] R. Wu, F. Blaabjerg, H. Wang, and M. Liserre, "Overview of catastrophic failures of freewheeling diodes in power electronic circuits" Microelectron. 
Reliab., Vol.53, pp.1788-1792, August, 2013.

[4] M. T. Rahimo, and N. Y. A. Shammas, "Freewheeling diode reverse-recovery failure modes in IGBT applications," IEEE Trans. Ind. Appl., Vol.37, pp.661-670, Mar, 2001.

[5] A. Amerasekera, Ch. Duvvury, W. Anderson, H. Gieser, and S. Ramaswamy, ESD in Silicon Integrated Circuits, Second Edition, John Wiley \& Sons, Ltd., England, 2002.

[6] J. Cambieri et al, "ESD induced leakage current increase of diffusion diodes," Proceeding of the 34th EOS/ESD Symposium, pp.1-6, September, 2012.

[7] F. Ch. Hou, G. Bosman, E. Simoen, J. Vanhellemont, and C. Claeys, IEEE Trans. Electron Devices. 45 (1998) 2528 - 2536.

[8] D. Bouangeune, et al, "ESD robustness of lowvoltage/high-speed TVS devices with epitaxial grown films," Proc. Of The 1st IEEE GCCE, pp.189-192, Oct., 2012.

[9] S. S. Choi, D. H. Cho and K. H. Shim, "Development of Transient Voltage Suppressor Device with Abrupt Junctions Embedded by Epitaxial Growth Technology," Electron. Mater. Lett. Vol.5, NO.2, pp. 59-62, June, 2009.

[10] D. Bouangeune et al, "Effect of electrostatic discharge on electrical properties of bidirectional TVS Zener diode with abrupt junction," Materials Transactions. Vol.54, pp.2125-2130, October, 2013.

[11] M. Miller, "Differences between Platinum- and gold-doped silicon power devices," IEEE Trans. Electron Devices ED. Vol.23, pp.1279-1283, December, 1976.

[12] D. E. Houston, M. S. Adler, and E. D. Wolley, " measurement and analysis of charge distribution and their decay in fast switching power rectifiers," International Electron Devices Meeting. Vol.23, pp.308-312, 1977.

[13] MSR1560-D, http://www.onsemi.com/pub/Collateral/MSR1560D.PDF.

[14] 15eth06, http://www.irf.com/product-

[15] info/datasheets/data/15eth06.pdf.

[16] Ch. Wang, L. Yin, and Ch. Wang, "A PhysicsBased Model for Fast Recovery Diodes with Lifetime Control and Emitter Efficiency Reduction," International Journal of Electronics and Electrical Engineering, Vol.6, pp.7-11 January, 2012.

[17] S.M. Sze, and Kwok k. NG, Physic of semiconductor device, 3 th Edition, John Wiley \& Sons Inc., Canada, pp. 281-304, 2007.

[18] A. Jahanzeb et al, “ TLP characterization for testing system level ESD performance," EOS/ESD Symposium, pp.1-8, October, 2010.

[19] G. Chen et al, "Sn enhanced TLP system for ESD characterization in semiconductor technologies," The 3rd ICCRD, pp.402-406, March, 2011.

[20] S.C. Huang et al, "Circuit and silicide impact on the correlation between TLP and ESD (HBM and MM)," IEEE International Integrated Reliability Workshop Final Report, pp.169-172, October, 2004.

[21] S H. Voldman et al, "Standardization of the transmission line pulse (TLP) methodology for electrostatic discharge (ESD)," EOS/ESD Symposium, pp.1-10, September, 2003.

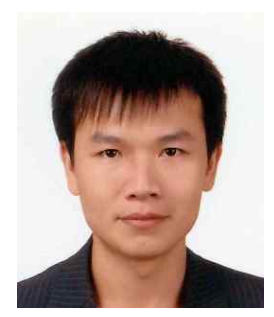

Daoheung Bouangeune was born in Sayaboury, Laos, on May 28, 1986. He received the B.S degree in physics from National University of Laos in2008 and M.S degree in applied physic from National University of Laos in 2011. He is currently working toward the $\mathrm{PhD}$ degree on semiconductor engineering at Chonbuk National University, Republic of Korea. His research interests in fabrication and developing ESD protection devices which relative to Zener diode, TVS diode, RC and LC filter.

Sang-Sik Choi is a senior researcher of Research and Development Division, Sigetronics, Inc., Jeonju 561-756, Republic of Korea.

Deok-Ho Cho is a director of Research and Development Division, Sigetronics, Inc., Jeonju 561-756, Republic of Korea. 


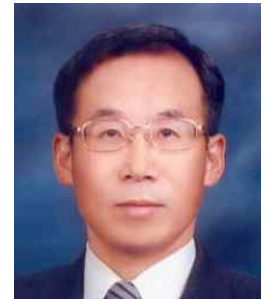

Kyu-Hwan Shim received his BS and MS degrees in materials science and engineering from Korea University in 1984 and 1986, respectively, and his $\mathrm{PhD}$ degree from the University of Illinois at Urbana-Champaign (UIUC).Meanwhile he joined the Electronics and Telecommunications Research Institute (ETRI) in 1986, where his major activities were focused on compound semiconductor processes and devices like GaAs MESFETs until 1992. Thanks to ETRI's program, he could study at UIUC for his PhD degree and specialize the epitaxial growth and device development of GaN based heterostructures. For five years after 1999, while working as a principal research member, his efforts were devoted to SiGe HBTs, BiCMOS integrated circuits, and strained-silicon MOSFETs. Then he moved to the Chonbuk National University (CBNU) to become a professor in 2004, and continued his research on SiGe and related semiconductor materials, processes, and devices in his lab Intelligent Semiconductor Research Lab (ISRL). At the same time, he started to serve as a CEO for a new lab-based venture company, Sigetronics Ltd. established inside the CBNU campus, where various semiconductor devices such as Zener diode(for LEDs), TVS, ULC-TVS, ESD/EMI Filter, SBD, FRD, PowerFETs have been developed and commercialized. His research is focused mostly on the epitaxial growth of germanium on silicon substrates (Ge-on-Si) and its application for future electrical and photonic devices including the beyond-CMOS and the emitters/receivers of optical signals for communications and image signal processing as well. He wants to assist for a new generation started on the basis of GaN, Ge, Si in every academic and industrial sectors.

Sung-Yong Chang is a senior researcher of Power Integrity Group, Korea Electric Power Research Institute (KEPRI), Daejeon 305-380, Republic of Korea

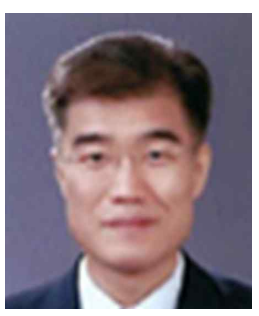

See-Jong Leem was Born in Korea, on December 23, 1960. He received his BS and MS degrees in materials science and engineering from Korea University in 1983 and 1987, respectively, and his $\mathrm{PhD}$ degree in electronic engineering from Korea University in 2001. He joined LG Electronic Institute of Technology in 1987, where his major activities were focused on compound semiconductor processes and devices like LD, LED, PD until 2006. He worked with HYOSUNG in 2007, where he was involved in R\&D, mass production on GaN based LED as senior vice president. Then he moved to the DONGSHIN university to become a professor in 2012. His research interests include the various semiconductor devices such as $\mathrm{GaN}$ based LED, LD, PD, OLED and solar cell.

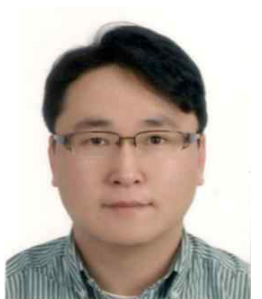

Chel-Jong Choi was born in Korea, on June 16, 1974. He received the B.S. degree in ceramic engineering from Hanyang University, Seoul, Republic of Korea, in 1997, and the M.S. and Ph.D. degrees in materials science engineering from the Gwangju Institute of Science and Technology (GIST), Gwangju, Republic of Korea, in 1999 and 2003, respectively. From 2003 to2005, he was with the Samsung Advanced Institute of Technology (SAIT), Suwon, Republic of Korea, in the areas of semiconductor-device characterization. From 2005 to2008, he worked with the Electronics and Telecommunications Research Institute (ETRI), Daejon, Republic of Korea, where he was involved in the process integration of nano-scaled Schottky barrier MOSFETs. Since 2008, he has been with Chonbuk National University, Jeonju, Republic of Korea. His research interests include the novel nanoscaled Ge- and III-Vbased electronic devices for the ultimate CMOS and post-CMOS technologies. 